\title{
Volatile Profile of Wall Rocket Baby-Leaves (Diplotaxis erucoides) Grown under Greenhouse: Main Compounds and Genotype Diversity
}

\author{
Carla Guijarro-Real ${ }^{\mathbb{D}}$, Adrián Rodríguez-Burruezo and Ana Fita * \\ Instituto de Conservación y Mejora de la Agrodiversidad Valenciana (COMAV), Universitat Politècnica \\ de València, 46022 Valencia, Spain; carguire@etsia.upv.es (C.G.-R.); adrodbur@upvnet.upv.es (A.R.-B.) \\ * Correspondence: anfifer@btc.upv.es; Tel.: +34-963-87-9418
}

Received: 7 May 2020; Accepted: 3 June 2020; Published: 5 June 2020

\begin{abstract}
Wall rocket is a leafy vegetable with pungent flavor related to the presence of isothiocyanates (ITCs). Despite interest in it as a crop of high organoleptic quality, the variability of the volatile profile in the species remains unknown. Twenty-four populations grown under a greenhouse were evaluated. A considerable diversity for the total levels of volatiles was found, providing information of the aroma intensity among accessions. ITCs represented the main fraction. Allyl ITC was the main compound, and levels showed up to 6-fold difference among populations. The esters fraction was mainly represented by cis-3-hexenyl isovalerate and cis-3-hexenyl butyrate, with 20-fold differences among populations. Additionally, the content in sinigrin was evaluated as main GSL in wall rocket. Differences reached up to 13-fold. These results suggest that some populations can be used to develop highly pungent varieties, whereas some others can be selected for mild-pungent varieties, as it is the case of DER045 with low levels of ITCs and high in esters. The presence of several ITCs in the profile also suggested the presence of other novel GSLs. Overall, the work increases the knowledge in the variability of wall rocket for the volatile profile and sinigrin accumulation, a starting point for future breeding programs.
\end{abstract}

Keywords: allyl isothiocyanate; Diplotaxis erucoides; esters; GC-MS; HS-SPME; new crops; sinigrin; variability; wall rocket

\section{Introduction}

Minor crops of the family Brassicaceae are highly appreciated in the cuisine, as they provide particular flavors to dishes like salads, pasta, pizza or meat. This group includes leafy vegetables such as watercress (Nasturtium officinale R. Br.), turnip greens (Brassica rapa L. subsp. rapa) and the rocket crops or arugula. Within the rocket germplasm, only the species Eruca sativa Mill. (salad rocket) and Diplotaxis tenuifolia (L.) DC. (wild rocket) have been established as crops of economic importance [1,2]. However, germplasm related to these species can be also considered of interest as potential crops [3], as it is the particular case of the edible wall rocket (Diplotaxis erucoides (L.) DC. subsp. erucoides) [4]. Wall rocket is an annual plant widespread in Central and Southern Europe, Northern Africa and Western Asia, and also naturalized in the template regions of America [5]. The tender leaves of this wild vegetable have been traditionally appreciated for their characteristic pungent and sulfurous flavor. Thus, in different Mediterranean countries, people would collect them for enriching soups, salads, omelettes and pasta dishes $[3,6,7]$. This popularity, combined with the adaptation of the species to the Mediterranean area, offers an opportunity for wall rocket to be established as a crop [4,8-10].

Despite the traditional interest in wall rocket and its potential to become a crop, the organoleptic information for the species is scarce. Guijarro-Real et al. [4] reported that the volatile profile of 
wall rocket was rich in isothiocyanates (ITCs), as previously described for rocket crops [11]. ITCs are nitrogen- and sulfur- containing compounds derived from the hydrolysis of their precursors, the glucosinolates (GSLs). Although GSLs can derive in several groups of compounds, ITCs are the main hydrolytic compounds obtained upon $\mathrm{pH}$ close to 7 and absence of ferrous ions and specific proteins [12]. In the particular case of wall rocket, allyl ITC has been reported as the main compound in the volatile profile of microgreens and seedlings [4]. This compound has been associated with the sensory descriptors of bitter taste, pungent, sulfurous, mustard-like, horseradish-like and lachrymose, as compiled by Bell et al. [13]. Consequently, its accumulation in high levels can be considered greatly responsible for the characteristic flavor of wall rocket. Furthermore, allyl ITC has been also investigated for its potential health benefits, mainly as antitumoral agent [14-16]. Therefore, including vegetables that are source of allyl ITC in our diet may have a positive effect in increasing health status.

The variability at the species level for allyl ITC and other possible novel ITCs in D. erucoides remains unknown, despite the contribution in the flavor attributes and potential nutritional quality of this vegetable. The evaluation of large germplasm collections is an essential starting point for future selection and breeding programs, as it provides information regarding the variability that could be found in the species. It may also allow identifying some promising accessions for the development of commercial varieties. We previously described the volatile profile of wall rocket in microgreens, seedlings and baby-leaves, based on one accession per phenological stage [4], but the intraspecies variability has not been previously studied. In this context, the present study was aimed at (1) evaluating the diversity of the profile of volatiles in a comprehensive set of wall rocket populations at the baby-leaf stage; (2) evaluating the accumulation of the GSL sinigrin in this set of materials, as it is the precursor of allyl ITC and has been previously reported as the main GSL in the species $[8,17,18]$; and (3) describing the co-occurrence of other minor GSLs. Results of this work will increase the knowledge in the species, and will also provide valuable information for current and future breeding programs, as it is a first insight of the variability in this species.

\section{Materials and Methods}

\subsection{Plant Material Cultivation and Preparation of Samples}

As plant material, twenty-four populations of wall rocket were used. The materials represented the first generation of wild populations collected along the Valencian Community (Spain) during the spring of 2015 (Table 1). These populations are conserved at the Universitat Politècnica de València as seeds (UPV, Valencia, Spain).

Materials were grown from May to the beginning of July 2016. Prior to sowing, seeds were treated with $2.5 \%(v / v)$ commercial bleach for $5 \mathrm{~min}$ followed by $100 \mathrm{ppm}$ gibberellic acid (Duchefa Biochemie, Haarlem, The Netherlands) solution for $24 \mathrm{~h}$ [19]. Seeds were then sown in individual seedling trays filled with commercial Neuhaus Humin-substrat N3 substrate (Klasmann-Deilmann Gmbh, Geeste, Germany), and placed under long day conditions $\left(16 / 8 \mathrm{~h}, \mathrm{~T}=25^{\circ} \mathrm{C}\right)$. One week later, the trays were moved to a greenhouse at the UPV experimental station $\left(39^{\circ} 28^{\prime} 60^{\prime \prime} \mathrm{N}, 0^{\circ} 20^{\prime} 27^{\prime \prime} \mathrm{W}\right)$. After two weeks, the seedlings were transplanted to larger pots $(15 \mathrm{~L})$ containing a mixture of coconut fiber (Horticoco, Valimex, Valencia, Spain) and N3 substrate (1:1). Three pots per population were transplanted, with 25 plants each. The plants were fertigated using a drip irrigation system, with the following final concentration for the main cations and anions in the watering: $11.47 \mathrm{mM} \mathrm{NO}_{3}{ }^{-}, 1.00 \mathrm{mM} \mathrm{NH}_{4}{ }^{+}$, $1.50 \mathrm{mM} \mathrm{H}_{2} \mathrm{PO}_{4}{ }^{-}, 6.75 \mathrm{mM} \mathrm{K}^{+}, 3.25 \mathrm{mM} \mathrm{Ca}^{2+}, 2.50 \mathrm{mM} \mathrm{Mg}^{2+}, 2.82 \mathrm{mM} \mathrm{SO}_{4}{ }^{2-}$; and the following salts: $50 \mu \mathrm{M} \mathrm{H}_{3} \mathrm{BO}_{3}, 10 \mu \mathrm{M}$ Fe-EDTA, $4.5 \mu \mathrm{M} \mathrm{MnCl}_{2}, 3.8 \mu \mathrm{M} \mathrm{ZnSO}_{4}, 0.3 \mu \mathrm{M} \mathrm{CuSO}_{4}, 0.1 \mu \mathrm{M}\left(\mathrm{NH}_{4}\right)_{6} \mathrm{Mo}_{7} \mathrm{O}_{24}$.

To prepare the samples, plants were harvested at the appearance of the first flower bud and before stem elongation [19], and only leaves without stems were used. While this wild vegetable is commonly harvested before flowering, taking the appearance of flower bud as reference allowed us to ensure the same phenological stage in all populations. For each population, the material from the different plants was harvested together, and split in two subsamples. One subsample was used for the analysis 
of the volatile organic compounds (VOCs) in fresh leaves. The second subsample was used for the analysis of sinigrin. In this case, the material was frozen at $-80^{\circ} \mathrm{C}$, freeze-dried and ground using a commercial grinder, then sealed with silica gel for control of humidity and stored in darkness until analysis. Three technical replicates were performed per population and analytical procedure.

Table 1. Geographical origin of the wild populations where the seeds were collected.

\begin{tabular}{|c|c|c|c|c|}
\hline \multirow{2}{*}{ Code } & \multirow{2}{*}{ Location } & \multirow{2}{*}{ Province } & \multicolumn{2}{|c|}{ Coordinates } \\
\hline & & & Latitude & Longitude \\
\hline DER089 & Cabanes & Castellón & $40^{\circ} 11^{\prime} 06^{\prime \prime} \mathrm{N}$ & $0^{\circ} 10^{\prime} 17^{\prime \prime} \mathrm{E}$ \\
\hline DER096 & Puebla-Tornesa & Castellón & $40^{\circ} 05^{\prime} 26^{\prime \prime} \mathrm{N}$ & $0^{\circ} 00^{\prime} 19^{\prime \prime} \mathrm{W}$ \\
\hline DER087 & Benicásim & Castellón & $40^{\circ} 02^{\prime} 49^{\prime \prime} \mathrm{N}$ & $0^{\circ} 03^{\prime} 19^{\prime \prime} \mathrm{E}$ \\
\hline DER069 & Aras de los Olmos & Valencia & $39^{\circ} 55^{\prime} 12^{\prime \prime} \mathrm{N}$ & $1^{\circ} 07^{\prime} 04^{\prime \prime} \mathrm{W}$ \\
\hline DER082 & Chilches & Castellón & $39^{\circ} 46^{\prime} 48^{\prime \prime} \mathrm{N}$ & $0^{\circ} 11^{\prime} 06^{\prime \prime} \mathrm{W}$ \\
\hline DER068 & Tuéjar & Valencia & $39^{\circ} 46^{\prime} 31^{\prime \prime} \mathrm{N}$ & $1^{\circ} 02^{\prime} 23^{\prime \prime} \mathrm{W}$ \\
\hline DER071 & Albalat dels Tarongers & Valencia & $39^{\circ} 41^{\prime} 60^{\prime \prime} \mathrm{N}$ & $0^{\circ} 20^{\prime} 01^{\prime \prime} \mathrm{W}$ \\
\hline DER064 & Casinos & Valencia & $39^{\circ} 41^{\prime} 49^{\prime \prime} \mathrm{N}$ & $0^{\circ} 42^{\prime} 49^{\prime \prime} \mathrm{W}$ \\
\hline DER067 & Losa del Obispo & Valencia & $39^{\circ} 41^{\prime} 48^{\prime \prime} \mathrm{N}$ & $0^{\circ} 53^{\prime} 18^{\prime \prime} \mathrm{W}$ \\
\hline DER062 & Liria & Valencia & $39^{\circ} 39^{\prime} 06^{\prime \prime} \mathrm{N}$ & $0^{\circ} 34^{\prime} 27^{\prime \prime} \mathrm{W}$ \\
\hline DER102 & Puzol & Valencia & $39^{\circ} 36^{\prime} 38^{\prime \prime} \mathrm{N}$ & $0^{\circ} 17^{\prime} 53^{\prime \prime} \mathrm{W}$ \\
\hline DER061 & La Eliana & Valencia & $39^{\circ} 32^{\prime} 59^{\prime \prime} \mathrm{N}$ & $0^{\circ} 31^{\prime} 18^{\prime \prime} \mathrm{W}$ \\
\hline DER060 & Paterna & Valencia & $39^{\circ} 32^{\prime} 13^{\prime \prime} \mathrm{N}$ & $0^{\circ} 26^{\prime} 54^{\prime \prime} \mathrm{W}$ \\
\hline DER103 & Pueblo Nuevo & Valencia & $39^{\circ} 31^{\prime} 19^{\prime \prime} \mathrm{N}$ & $0^{\circ} 23^{\prime} 17^{\prime \prime} \mathrm{W}$ \\
\hline DER056 & Pueblo Nuevo & Valencia & $39^{\circ} 30^{\prime} 39^{\prime \prime} \mathrm{N}$ & $0^{\circ} 22^{\prime} 57^{\prime \prime} \mathrm{W}$ \\
\hline DER057 & Valencia & Valencia & $39^{\circ} 29^{\prime} 04^{\prime \prime} \mathrm{N}$ & $0^{\circ} 24^{\prime} 19^{\prime \prime} \mathrm{W}$ \\
\hline DER052 & Sollana & Valencia & $39^{\circ} 16^{\prime} 18^{\prime \prime} \mathrm{N}$ & $0^{\circ} 22^{\prime} 55^{\prime \prime} \mathrm{W}$ \\
\hline DER039 & Játiva & Valencia & $38^{\circ} 58^{\prime} 41^{\prime \prime} \mathrm{N}$ & $0^{\circ} 30^{\prime} 53^{\prime \prime} \mathrm{W}$ \\
\hline DER040 & Bellús & Valencia & $38^{\circ} 56^{\prime} 38^{\prime \prime} \mathrm{N}$ & $0^{\circ} 29^{\prime} 23^{\prime \prime} \mathrm{W}$ \\
\hline DER006 & Oliva & Valencia & $38^{\circ} 54^{\prime} 42^{\prime \prime} \mathrm{N}$ & $0^{\circ} 06^{\prime} 49^{\prime \prime} \mathrm{W}$ \\
\hline DER008 & Javea & Alicante & $38^{\circ} 47^{\prime} 35^{\prime \prime} \mathrm{N}$ & $0^{\circ} 06^{\prime} 41^{\prime \prime} \mathrm{E}$ \\
\hline DER045 & Jijona & Alicante & $38^{\circ} 38^{\prime} 30^{\prime \prime} \mathrm{N}$ & $0^{\circ} 28^{\prime} 37^{\prime \prime} \mathrm{W}$ \\
\hline DER021 & Aspe & Alicante & $38^{\circ} 18^{\prime} 33^{\prime \prime} \mathrm{N}$ & $0^{\circ} 47^{\prime} 35^{\prime \prime} \mathrm{W}$ \\
\hline DER014 & Santa Pola & Alicante & $38^{\circ} 14^{\prime} 36^{\prime \prime} \mathrm{N}$ & $0^{\circ} 32^{\prime} 17^{\prime \prime} \mathrm{W}$ \\
\hline
\end{tabular}

\subsection{Extraction and Analysis of Volatile Organic Compounds}

The subsamples used for the analysis of VOCs were stored at $4{ }^{\circ} \mathrm{C}$ in sealed plastic bags after harvesting and analyzed within $24 \mathrm{~h}$. The extraction of VOCs was performed by headspace-solid phase microextraction (HS-SPME) and analyzed by gas chromatography-mass spectrometry (GC-MS). We followed the protocol described in Guijarro-Real et al. [20]. Briefly, $1.5 \mathrm{~g}$ of fresh material were chopped in slices of approximately $0.5 \mathrm{~cm}$ long and immediately placed into $20 \mathrm{~mL}$ sealed headspace vials. The samples were pre-incubated at $40{ }^{\circ} \mathrm{C}$ for $30 \mathrm{~min}$, and then incubated with the fiber $(50 / 30 \mu \mathrm{m}$ DVB/CAR/PDMS; Supelco, Bellefonte, PA, USA) for another $40 \mathrm{~min}$ at the same temperature to allow the adsorption of volatiles. The thermal desorption took place at $250{ }^{\circ} \mathrm{C}$ for $30 \mathrm{~s}$ (splitless mode). The fiber was conditioned at $270{ }^{\circ} \mathrm{C}$ for $1 \mathrm{~h}$ prior to the first analysis and reconditioned at $250{ }^{\circ} \mathrm{C}$ for 30 min between samples.

A 6890 N Network GC System with autosampler, coupled to a 5973 Inert Mass Selective Detector (Agilent Technologies) and equipped with a HO-5MS J\&W silica capillary column (5\% phenyl-95\% methylpolysiloxane, $30 \mathrm{~m} \times 0.25 \mathrm{~mm}$ i.d., $0.25 \mu \mathrm{m}$ thickness film; Agilent Technologies), was used for the analysis of VOCs. The following thermal gradient was programmed: increasing from $100{ }^{\circ} \mathrm{C}$ to $250^{\circ} \mathrm{C}$ in $30 \mathrm{~min}$, then stabilized at $250^{\circ} \mathrm{C}$ for $10 \mathrm{~min}$. The transfer line was maintained at $220^{\circ} \mathrm{C}$. Helium was used as a carrier gas at a constant flow of $1 \mathrm{~mL} \mathrm{~min}^{-1}$. The detection by the mass spectrometer was performed with the electron impact mode (70 eV ionization energy, source temperature $\left.225^{\circ} \mathrm{C}\right)$, with the acquisition performed in the scanning mode (mass range m/z 35-350 amu). The MSD ChemStation D.02.00.275 (Agilent Technologies) was used to process the chromatograms and spectra. A tentative 
identification was established by comparing the obtained mass spectra with the NIST 2005 Mass Spectral Library, and also by comparing the GC retention times, mass spectra and estimated retention indices in our customized library [21]. As identification was tentative, only those compounds scoring above $80 \%$ match with the identified NIST mass spectra were considered to be positive identification [22]. Finally, the levels of VOCs were semi-quantified based on the integration of GC peak areas from the total ion current chromatograms $[20,21]$.

\subsection{Extraction and Analysis of Sinigrin}

The content in sinigrin was analyzed as described in Guijarro-Real et al. [8]. The analysis was performed with freeze-dried material. Materials were weighed before (fresh weight, FW) and after (dry weight, DW) the freeze-drying process. The difference between both weights was used to determine the percentages in humidity for the leaves according to the formula:

$$
\% \text { humidity }=\frac{F W-D W}{F W} \times 100
$$

For the extraction and analysis, $0.1 \mathrm{~g}$ of the dried ground material were heated for $2 \mathrm{~min}$ at $75^{\circ} \mathrm{C}$ to inactivate the plant myrosinase [23]. Then, $1 \mathrm{~mL}$ of methanol $70 \%(v / v)$ was added and the mixture was incubated for $15 \mathrm{~min}$ at $75^{\circ} \mathrm{C}$ in a Thermoblock TD150 P2 (Falc Instruments, Treviglio, Italy). After the centrifugation of the samples (4,500 rpm for $5 \mathrm{~min}$ ), the supernatant was collected ant the extraction step repeated. Supernatants were injected into SPE columns containing a DEAE Sephadex anion exchanger (A-25; Sigma-Aldrich, Saint Louis, MO, USA), which were previously activated with $20 \mathrm{mM}$ sodium acetate buffer ( $\mathrm{pH}$ 5.5).

The extracted glucosinolates were incubated with $20 \mu \mathrm{L}$ of diluted aryl sulfatase (type $\mathrm{H}-1$ from Helix pomatia; Sigma-Aldrich) overnight. The desulfo-glucosinolates were eluted with $1 \mathrm{~mL}$ of milliQ water. The analysis was performed using an HPLC 1220 Infinity LC System (Agilent Technologies, Santa Clara, CA, USA) with a Luna ${ }^{\circledR}$ Omega $C_{18}$ column $(150 \mathrm{~mm} \times 4.6 \mathrm{~mm}$ i.d., $3 \mu \mathrm{m}$ particle size; Phenomex, Torrance, CA, USA). As mobile phase, acetonitrile (A) and water (B) were used with the following gradient: from $98 \%$ A to $65 \%$ in $35 \mathrm{~min}$ and then equilibrated to the initial conditions for $5 \mathrm{~min}$, at a flow rate of $0.75 \mathrm{~mL} \mathrm{~min}^{-1}$. The injection volume was $10 \mu \mathrm{L}$, and the signal was recorded at $229 \mathrm{~nm}$. Sinigrin hydrate (PhytoPlan, Heidelberg, Germany) was used as external standard for the quantification after desulfation. Results were expressed as $\mathrm{mg}$ of sinigrin in each $100 \mathrm{~g}$ of fresh leaves (mg $100 \mathrm{~g}^{-1} \mathrm{FW}$ ).

\subsection{Statistical Analysis}

Three replicates were used to obtain the mean values for the levels of VOCs and the content in sinigrin. Relative abundances for individual VOCs were also calculated as the ratio between the GC-peak area against the added area of the total of volatiles identified and expressed as percentage [20]. Data were $\log _{2}$-transformed for normalization and subjected to a one-way factorial Analysis of Variance (ANOVA). Significant differences were assessed by the multiple range Student-Newman-Keuls test $(p=0.05)$. The Statgraphics Centurion XVII software was used for the statistics.

$\log _{2}$-transformed data were also subjected to Principal Component Analysis (PCA) and Hierarchical Cluster Analysis by means of the web CustVis Tool developed with the R package version 0.10.2.1 for $\mathrm{R}$ statistics software [24]. For calculations, unit variance scaling was applied, the distance metrics based on the Pearson correlation and compounds clustered according to the average linkage [25]. 


\section{Results and Discussion}

\subsection{Profile in VOCs}

\subsubsection{Chemical Groups of Volatiles}

The volatile organic compounds were directly extracted from fresh materials using the headspace-solid phase microextraction technique (HS-SPME). The extraction of VOCs without the addition of solvents has been successfully used in plant materials including leaves such as salad rocket, water celery and parsley [20,22,26]. In addition, the HS-SPME technique was previously applied to several plant materials such as leaves and fruits [21,25-27]. Overall, the methodology used (i.e., the extraction of VOCs from the materials and tentative identification according to mass spectra match) was adequate to analyze the main compounds in wall rocket chromatograms.

The populations showed significant differences in their total levels of volatiles identified (Table 2). Thus, there was a considerable diversity in this trait, with several accessions showing total levels below $500 \times 10^{6}$ (GC-peak area units), such as DER014, DER045 and DER068, while others like DER039, DER056, DER057, DER087, DER089 and DER103 showed levels higher than $1000 \times 10^{6}$. Further analyses combining olfactometry tests to study the aroma of these materials (e.g., sniffing port) could clarify whether these differences might correlate with differences in the aroma intensity, which would be probably due to a combination of intensity and relative abundances of some individual VOCs [28].

Table 2. Absolute levels (GC-peak area units, $\times 10^{6}$ ) for the groups of volatile organic compounds identified in the wall rocket materials $(n=3)$. The relative abundances $(\%$, in parentheses) are also included.

\begin{tabular}{|c|c|c|c|c|}
\hline & Isothiocyanates & Esters & Others & Total \\
\hline DER006 & $819.9^{c}(81.1)$ & $87.5^{\mathrm{a}}(8.6)$ & $103.6^{\mathrm{d}}(10.3)$ & $1011.0^{\mathrm{bc}}$ \\
\hline DER008 & $741.2^{\mathrm{c}}(79.1)$ & $112.4^{\mathrm{a}}(12.0)$ & $83.1^{\mathrm{a}-\mathrm{d}}(8.9)$ & $936.7^{b c}$ \\
\hline DER014 & $340.7^{b c}(64.4)$ & $148.9^{\mathrm{a}}(28.2)$ & $39.2^{\mathrm{a}-\mathrm{d}}(7.4)$ & $528.8^{\mathrm{ab}}$ \\
\hline DER021 & $456.9^{b c}(71.3)$ & $96.0^{\mathrm{a}}(15.0)$ & $87.9^{\mathrm{bcd}}(13.7)$ & $640.7^{\mathrm{abc}}$ \\
\hline DER039 & $1041.2^{\mathrm{C}}(86.8)$ & $88.8^{\mathrm{a}}(7.4)$ & $68.9^{\mathrm{a}-\mathrm{d}}(5.8)$ & $1198.9^{c}$ \\
\hline DER040 & $413.0^{\mathrm{bc}}(59.7)$ & $206.5^{\mathrm{a}}(29.9)$ & $72.1^{\mathrm{a}-\mathrm{d}}(10.4)$ & $691.5^{\mathrm{bc}}$ \\
\hline DER045 & $186.2^{\mathrm{a}}(45.9)$ & $182.2^{\mathrm{a}}(44.9)$ & $37.1^{\mathrm{a}}(9.2)$ & $405.6^{\mathrm{a}}$ \\
\hline DER052 & $715.4^{\mathrm{c}}(79.7)$ & $145.2^{\mathrm{a}}(16.2)$ & $37.0^{\mathrm{a}-\mathrm{d}}(4.1)$ & $897.7^{\mathrm{bc}}$ \\
\hline DER056 & $1102.5^{\mathrm{C}}(88.9)$ & $75.7^{\mathrm{a}}(6.1)$ & $62.2^{\mathrm{a}-\mathrm{d}}(5.0)$ & $1240.4^{\mathrm{C}}$ \\
\hline DER057 & $1006.0^{\mathrm{C}}(87.8)$ & $74.9^{\mathrm{a}}(6.5)$ & $65.6^{\mathrm{a}-\mathrm{d}}(5.7)$ & $1146.5^{\mathrm{c}}$ \\
\hline DER060 & $882.9^{c}(85.5)$ & $81.5^{\mathrm{a}}(7.9)$ & $68.6^{\mathrm{a}-\mathrm{d}}(6.6)$ & $1033.0^{\mathrm{bc}}$ \\
\hline DER061 & $906.2^{\mathrm{c}}(87.4)$ & $88.9^{\mathrm{a}}(8.6)$ & $41.4^{\mathrm{a}-\mathrm{d}}(4.0)$ & $1036.6^{\mathrm{bc}}$ \\
\hline DER062 & $630.1^{\mathrm{bc}}(74.6)$ & $166.6^{\mathrm{a}}(19.7)$ & $48.4^{\mathrm{a}-\mathrm{d}}(5.7)$ & $845.0^{\mathrm{bc}}$ \\
\hline DER064 & $729.9^{c}(75.1)$ & $143.7^{\mathrm{a}}(14.8)$ & $98.7^{\mathrm{cd}}(10.1)$ & $972.3^{b c}$ \\
\hline DER067 & $722.5^{\mathrm{c}}(83.5)$ & $112.4^{\mathrm{a}}(13.0)$ & $30.3^{\mathrm{a}-\mathrm{d}}(3.5)$ & $865.2^{\mathrm{bc}}$ \\
\hline DER068 & $230.8^{\mathrm{b}}(45.3)$ & $248.2^{\mathrm{a}}(48.7)$ & $30.7^{a-d}(6.0)$ & $509.7^{\mathrm{ab}}$ \\
\hline DER069 & $500.8^{\mathrm{bc}}(75.3)$ & $139.4^{\mathrm{a}}(20.9)$ & $25.3^{\mathrm{abc}}(3.8)$ & $665.5^{\mathrm{bc}}$ \\
\hline DER071 & $498.5^{\mathrm{bc}}(82.3)$ & $60.3^{a}(9.9)$ & $47.0^{\mathrm{a}-\mathrm{d}}(7.8)$ & $605.8^{a b c}$ \\
\hline DER082 & $647.4^{\mathrm{c}}(81.7)$ & $122.1^{\mathrm{a}}(15.4)$ & $23.0^{\mathrm{abc}}(2.9)$ & $792.5^{\mathrm{bc}}$ \\
\hline DER087 & $909.1^{\mathrm{c}}(85.2)$ & $62.2^{\mathrm{a}}(5.8)$ & $96.1^{\mathrm{bcd}}(9.0)$ & $1067.4^{\mathrm{bc}}$ \\
\hline DER089 & $1097.6^{\mathrm{C}}(91.1)$ & $58.8^{\mathrm{a}}(4.9)$ & $48.6^{\mathrm{a}-\mathrm{d}}(4.0)$ & $1205.1^{\mathrm{c}}$ \\
\hline DER096 & $696.8^{c}(81.5)$ & $84.5^{\mathrm{a}}(9.9)$ & $74.0^{\mathrm{a}-\mathrm{d}}(8.6)$ & $855.4^{\mathrm{bc}}$ \\
\hline DER102 & $674.4^{\mathrm{c}}(72.5)$ & $138.1^{\mathrm{a}}(14.9)$ & $117.3^{\mathrm{d}}(12.6)$ & $929.8^{b c}$ \\
\hline DER103 & $938.0^{c}(91.4)$ & $66.4^{\mathrm{a}}(6.5)$ & $21.6^{\mathrm{ab}}(2.1)$ & $1026.0^{\mathrm{bc}}$ \\
\hline Mean & 703.7 (80.0) & $116.3(13.2)$ & $59.5(6.8)$ & 879.5 \\
\hline
\end{tabular}

Different letters within columns indicate significant differences between means according to the Student-NewmanKeuls test $(p=0.05)$.

Overall, accessions were rich in isothiocyanates (ITCs, on average $703.7 \times 10^{6}$ GC-peak area units) and esters $\left(116.3 \times 10^{6}\right)$ (Table 2). Interestingly, differences in the total level of volatiles as reported above were mainly due to significant differences in the levels of ITCs among accessions 
but not for the esters fraction. Indeed, the group of esters added between $66.4 \times 10^{6}$ (DER103) and $248.2 \times 10^{6}$ (DER068) GC-peak area units, with no significant differences among accessions due to the high intra-population variability. By contrast, there were significant differences between populations for the levels of ITCs, with DER045 showing the lowest level and DER056 the highest level, with an almost 6-fold increase.

Sulfur compounds are commonly reported within the Brassicaceae [22,29-32]. In particular, they have been identified as the main fraction in the volatile profile of wall rocket microgreens and seedlings [4] and in stems, leaves and pods of wild, mature plants [33]. By contrast, the families of aldehydes and alcohols were not greatly represented in the current study, while they have been reported in other species like the related rocket crops [22,33]. Instead, 3-hexenol derived esters were largely quantified by Guijarro-Real et al. [4] in seedlings and baby-leaves of wall rocket, while none of these families of volatiles were relevant according to Clemente-Villalba et al. [33]. Due to the qualitative differences among species and works, we consider that further studies should be addressed to understand whether it is related to the species, and if other factors affecting the volatile profile also influence.

In addition to total levels, populations also varied in the relative abundance of these groups (Table 2). Hence, although most of the populations showed percentages for ITCs above $70 \%$, some of them significantly decreased below 50\% like populations DER045 and DER068. In the same way, despite the average relative abundance of esters was $13.2 \%$, the percentages ranged from $4.9 \%$ (DER089) to $44.9 \%$ (DER045). The percentage of ITCs and other GSL-derived compounds previously reported in crops like salad rocket [22] or radish [31] is remarkably lower (below $6 \%$ but increasing up to $37 \%$ in radish roots), while aldehydes, alcohols and the derived esters have been found in higher abundances. Relative abundances of families of VOCs, and more particular, of individual compounds, affect the perception of the whole aroma. Thus, our results suggest that wall rocket may be of greater pungency than other related crops, as the species showed a high abundance in ITCs which are related to bitterness, pungency and lachrymose aroma [13].

Finally, a miscellany of four compounds was identified and included the alcohol cis-3-hexen-1-ol $(\mathrm{O} 1)$, the aldehyde decanal $(\mathrm{O} 2)$, the aliphatic hydrocarbon tetradecane $(\mathrm{O} 3)$ and the norcarotenoid $\beta$-ionone (O4) (Table 3). The percentage of this group was on average $6.8 \%$, with the alcohol as the main representative (Tables 2 and 4 ).

Table 3. List of volatile organic compounds (VOCs) identified in the present study including their chemical group and code assigned. The corresponding glucosinolate (GSL) from which each isothiocyanate derives is also indicated.

\begin{tabular}{lllll}
\hline Code & RI $^{\mathbf{a}}$ & VOC & Group $^{\mathbf{b}}$ & GSL \\
\hline I1 & 846 & allyl isothiocyanate & Itc & 2-propenyl GSL (sinigrin) \\
I2 & 951 & 3-butenyl isothiocyanate & Itc & 3-butenyl GSL (gluconapin) \\
I3 & 1041 & 3-methylbutyl isothiocyanate & Itc & 3-methylbutyl GSL \\
I4 & 1077 & pentyl isothiocyanate & Itc & pentyl GSL \\
I5 & 1185 & hexyl isothiocyanate & Itc & hexyl GSL \\
I6 & 1318 & benzyl isothiocyanate & Itc & benzyl GSL (glucotropaeolin) \\
I7 & 1429 & phenylethyl isothiocyanate & Itc & phenylethyl GSL (gluconasturtiin) \\
E1 & 1091 & cis-3-hexenyl propionate & Est & \\
E2 & 1183 & hexyl butyrate & Est & \\
E3 & 1191 & cis-3-hexenyl butyrate & Est & \\
E4 & 1226 & cis-3-hexenyl isovalerate & Est & \\
E5 & 1290 & cis-3-hexenyl valerate & Est & \\
O1 & 868 & cis-3-hexen-1-ol & Alc & \\
O2 & 1204 & Decanal & Ald & \\
O3 & 1413 & Tetradecane & Hyd & \\
O4 & 1457 & B-ionone & Norc & \\
\hline
\end{tabular}

${ }^{\mathrm{a} R I}$ : Retention index based on NIST2005. ${ }^{\mathrm{b}}$ Alc: Alcohol; Ald: Aldehyde; Est: Ester; Hyd: Aliphatic hydrocarbon; Itc: Isothiocyanate; Norc: Norcarotenoid. 
Table 4. Mean values (GC-peak area units, $\left.\times 10^{6}\right)$ for the individual VOCs identified in the wall rocket materials $(n=3)$. The relative abundances $(\%$, in parentheses) are also included.

\begin{tabular}{|c|c|c|c|c|c|c|c|c|c|c|c|c|c|c|c|c|}
\hline & I1 & I2 & I3 & I4 & I5 & I6 & I7 & E1 & E2 & E3 & E4 & E5 & 01 & $\mathrm{O} 2$ & $\mathrm{O} 3$ & $\mathrm{O} 4$ \\
\hline DER006 & $793.8^{c}(78.5)$ & $5.7^{\mathrm{b}}(0.6)$ & $12.9^{\mathrm{bc}}(1.3)$ & $\operatorname{tr}$ & $0.6^{\mathrm{a}}(0.1)$ & $4.7^{\mathrm{b}}(0.5)$ & $2.1^{\mathrm{b}}(0.2)$ & $8.8^{\mathrm{a}}(0.9)$ & $1.3^{\mathrm{a}}(0.1)$ & $37.7^{\mathrm{ab}}(3.7)$ & $34.0^{c-f}(3.4)$ & $5.6^{\mathrm{a}-\mathrm{e}}(0.6)$ & $99.1^{\mathrm{bc}}(9.8)$ & $0.4^{\mathrm{b}}(0.0)$ & $2.9^{\mathrm{b}}(0.3)$ & $1.2^{\mathrm{b}}(0.1)$ \\
\hline DER008 & $722.8^{\mathrm{c}}(77.2)$ & $3.4^{\mathrm{b}}(0.4)$ & $7.3^{\mathrm{bc}}(0.8)$ & $\operatorname{tr}$ & $0.6^{\mathrm{a}}(0.1)$ & $6.6^{\mathrm{b}}(0.7)$ & $0.7^{\mathrm{b}}(0.1)$ & $3.9^{\mathrm{a}}(0.4)$ & $1.1^{\mathrm{a}}(0.1)$ & $29.8^{\mathrm{ab}}(3.2)$ & $65.4^{\mathrm{d}-\mathrm{g}}(7.0)$ & $12.1^{\mathrm{d}-\mathrm{f}}(1.3)$ & $80.5^{b c}(8.6)$ & $0.3^{\mathrm{b}}(0.0)$ & $1.7^{b}(0.2)$ & $0.5^{\mathrm{b}}(0.1)$ \\
\hline DER014 & $326.1^{\mathrm{bc}}(61.7)$ & $6.1^{\mathrm{b}}(1.2)$ & $7.3^{\mathrm{bc}}(1.4)$ & - & $0.3^{\mathrm{a}}(0.0)$ & $0.6^{\mathrm{ab}}(0.1)$ & $0.4^{\mathrm{ab}}(0.1)$ & $6.4^{\mathrm{a}}(1.2)$ & $0.3^{\mathrm{a}}(0.1)$ & $23.5^{\mathrm{ab}}(4.4)$ & $108.4^{\mathrm{e}-\mathrm{g}}(20.5)$ & $10.3^{\mathrm{c}-\mathrm{f}}(1.9)$ & $35.9^{\mathrm{abc}}(6.8)$ & $0.2^{\mathrm{b}}(0.0)$ & $2.8^{\mathrm{b}}(0.5)$ & $0.3^{\mathrm{b}}(0.1)$ \\
\hline DER021 & $435.2^{\mathrm{bc}}(67.9)$ & $5.7^{\mathrm{b}}(0.9)$ & $12.2^{\mathrm{bcc}}(1.9)$ & $\operatorname{tr}$ & $0.8^{\mathrm{a}}(0.1)$ & $2.3^{\mathrm{b}}(0.4)$ & $0.7^{\mathrm{b}}(0.1)$ & $13.7^{\mathrm{a}}(2.1)$ & $0.6^{\mathrm{a}}(0.1)$ & $27.5^{\mathrm{ab}}(4.3)$ & $46.0^{\mathrm{d}-\mathrm{g}}(7.2)$ & $8.0^{\mathrm{b}-\mathrm{f}}(1.3)$ & $84.8^{\mathrm{bc}}(13.2)$ & $0.3^{\mathrm{b}}(0.0)$ & $2.2^{\mathrm{b}}(0.3)$ & $0.6^{\mathrm{b}}(0.1)$ \\
\hline DER039 & $1007.3^{\mathrm{C}}(84.0)$ & $9.2^{\mathrm{b}}(0.8)$ & $14.5^{\mathrm{bc}}(1.2)$ & $\operatorname{tr}$ & $0.5^{\mathrm{a}}(0.0)$ & $5.6^{\mathrm{b}}(0.4)$ & $4.5^{\mathrm{b}}(0.4)$ & $12.0^{\mathrm{a}}(1.0)$ & $1.4^{\mathrm{a}}(0.1)$ & $61.2^{\mathrm{ab}}(5.1)$ & $11.1^{\mathrm{a}-\mathrm{c}}(0.9)$ & $3.0^{\mathrm{ab}}(0.2)$ & $65.4^{\mathrm{bc}}(5.5)$ & $0.4^{\mathrm{b}}(0.0)$ & $1.9^{\mathrm{b}}(0.2)$ & $1.3^{\mathrm{b}}(0.1)$ \\
\hline DER040 & $398.6^{\mathrm{bc}}(57.6)$ & $3.2^{\mathrm{b}}(0.5)$ & $9.8^{\mathrm{bc}}(1.4)$ & - & - & $0.7^{\mathrm{b}}(0.1)$ & $0.7^{\mathrm{b}}(0.1)$ & $26.2^{\mathrm{a}}(3.8)$ & $1.7^{\mathrm{a}}(0.2)$ & $98.2^{\mathrm{b}}(14.2)$ & $72.5^{\mathrm{d}-\mathrm{g}}(10.5)$ & $8.0^{b-f}(1.2)$ & $70.6^{\mathrm{bc}}(10.2)$ & $0.2^{\mathrm{b}}(0.0)$ & $1.1^{\mathrm{ab}}(0.2)$ & $0.2^{\mathrm{b}}(0.0)$ \\
\hline DER045 & $182.8^{\mathrm{a}}(45.1)$ & $1.5^{\mathrm{a}}(0.4)$ & $1.8^{\mathrm{a}}(0.5)$ & - & $\operatorname{tr}$ & - & - & $5.9^{\mathrm{a}}(1.4)$ & $0.2^{\mathrm{a}}(0.0)$ & $13.4^{\mathrm{ab}}(3.3)$ & $146.6^{\mathrm{g}}(36.1)$ & $16.2^{\mathrm{ef}}(4.0)$ & $30.4^{\mathrm{a}}(7.5)$ & $0.2^{\mathrm{b}}(0.1)$ & $6.3^{\mathrm{b}}(1.5)$ & $0.2^{\mathrm{ab}}(0.1)$ \\
\hline DER052 & $696.6^{\mathrm{c}}(77.6)$ & $5.1^{\mathrm{b}}(0.6)$ & $10.4^{\mathrm{bc}}(1.2)$ & $\operatorname{tr}$ & $0.3^{\mathrm{a}}(0.0)$ & $1.3^{\mathrm{b}}(0.1)$ & $1.8^{\mathrm{b}}(0.2)$ & $10.3^{\mathrm{a}}(1.1)$ & $1.5^{\mathrm{a}}(0.2)$ & $89.4^{\mathrm{b}}(10.0)$ & $38.7^{\mathrm{cg} g}(4.3)$ & $5.3^{a}-\mathrm{e}(0.6)$ & $35.0^{\mathrm{abc}}(3.9)$ & $0.1^{\mathrm{b}}(0.0)$ & $1.7^{\mathrm{b}}(0.2)$ & $0.1^{\mathrm{b}}(0.0)$ \\
\hline DER056 & $1065.2^{\mathrm{c}}(85.9)$ & $10.9^{\mathrm{b}}(0.9)$ & $16.8^{\mathrm{bc}}(1.4)$ & $\operatorname{tr}$ & $0.8^{\mathrm{a}}(0.1)$ & $6.8^{\mathrm{b}}(0.5)$ & $1.9^{\mathrm{b}}(0.2)$ & $7.0^{\mathrm{a}}(0.6)$ & $1.0^{\mathrm{a}}(0.1)$ & $32.5^{\mathrm{ab}}(2.6)$ & $31.8^{\mathrm{b}-\mathrm{e}}(2.6)$ & $3.3^{\mathrm{a}-\mathrm{c}}(0.3)$ & $58.2^{\mathrm{abc}}(4.7)$ & $0.2^{\mathrm{b}}(0.0)$ & $2.4^{\mathrm{b}}(0.2)$ & $1.4^{\mathrm{b}}(0.1)$ \\
\hline DER057 & $980.5^{c}(85.5)$ & $7.1^{\mathrm{b}}(0.6)$ & $10.3^{\mathrm{bc}}(0.9)$ & 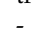 & $0.5^{\mathrm{a}}(0.0)$ & $4.1^{\mathrm{b}}(0.4)$ & $3.6^{\mathrm{b}}(0.3)$ & $8.3^{\mathrm{a}}(0.7)$ & $1.3^{\mathrm{a}}(0.1)$ & $55.6^{\mathrm{ab}}(4.8)$ & $7.8^{\mathrm{a}}(0.7)$ & $1.9^{\mathrm{a}}(0.2)$ & $60.8^{\mathrm{abc}}(5.3)$ & $0.2^{\mathrm{b}}(0.0)$ & $3.5^{\mathrm{b}}(0.3)$ & $1.1^{\mathrm{b}}(0.1)$ \\
\hline DER060 & $852.9^{c}(82.6)$ & $9.2^{\mathrm{b}}(0.9)$ & $16.5^{\mathrm{bc}}(1.6)$ & $\operatorname{tr}$ & $0.3^{\mathrm{a}}(0.0)$ & $3.2^{\mathrm{b}}(0.3)$ & $0.6^{\mathrm{b}}(0.1)$ & $7.7^{\mathrm{a}}(0.7)$ & $0.9^{\mathrm{a}}(0.1)$ & $43.0^{\mathrm{ab}}(4.2)$ & $25.3^{\mathrm{b}-\mathrm{d}}(2.5)$ & $4.6^{\mathrm{a}-\mathrm{d}}(0.4)$ & $67.3^{\mathrm{bc}}(6.5)$ & $0.2^{\mathrm{b}}(0.0)$ & $0.2^{\mathrm{a}}(0.0)$ & $0.9^{\mathrm{b}}(0.1)$ \\
\hline DER061 & $881.1^{\mathrm{c}}(85.0)$ & $10.5^{\mathrm{b}}(1.0)$ & $11.3^{\mathrm{bc}}(1.1)$ & $\operatorname{tr}$ & $0.3^{\mathrm{a}}(0.0)$ & $2.0^{\mathrm{ab}}(0.2)$ & $1.0^{\mathrm{b}}(0.1)$ & $7.4^{\mathrm{a}}(0.7)$ & $1.4^{\mathrm{a}}(0.1)$ & $51.9^{\mathrm{ab}}(5.0)$ & $25.1^{\mathrm{b}-\mathrm{d}}(2.4)$ & $3.3^{\mathrm{a}-\mathrm{c}}(0.3)$ & $38.1^{\mathrm{abc}}(3.7)$ & $0.2^{\mathrm{b}}(0.0)$ & $2.7^{\mathrm{b}}(0.3)$ & $0.5^{\mathrm{b}}(0.0)$ \\
\hline DER062 & $609.8^{\mathrm{bc}}(72.2)$ & $5.0^{\mathrm{b}}(0.6)$ & $12.7^{\mathrm{bc}}(1.5)$ & - & $0.3^{\mathrm{a}}(0.0)$ & $1.4^{\mathrm{b}}(0.2)$ & $0.9^{\mathrm{b}}(0.1)$ & $15.0^{\mathrm{a}}(1.8)$ & $0.8^{\mathrm{a}}(0.1)$ & $44.8^{\mathrm{ab}}(5.3)$ & $92.6^{\mathrm{d}-\mathrm{g}}(11.0)$ & $13.3^{\mathrm{d}-\mathrm{f}}(1.6)$ & $46.1^{\mathrm{abc}}(5.5)$ & $0.2^{\mathrm{b}}(0.0)$ & $1.8^{\mathrm{b}}(0.2)$ & $0.3^{\mathrm{b}}(0.0)$ \\
\hline DER064 & $702.9^{c}(72.3)$ & $6.4^{\mathrm{b}}(0.7)$ & $12.5^{\mathrm{bc}}(1.3)$ & $\operatorname{tr}$ & $0.8^{\mathrm{a}}(0.1)$ & $5.4^{\mathrm{b}}(0.6)$ & $1.9^{\mathrm{b}}(0.2)$ & $19.0^{\mathrm{a}}(2.0)$ & $1.6^{\mathrm{a}}(0.2)$ & $60.5^{\mathrm{ab}}(6.2)$ & $55.2^{\mathrm{d}-\mathrm{g}}(5.7)$ & $7.4^{\mathrm{b}-\mathrm{f}}(0.8)$ & $95.9^{\mathrm{bc}}(9.9)$ & $0.3^{\mathrm{b}}(0.0)$ & $2.0^{\mathrm{b}}(0.2)$ & $0.4^{\mathrm{b}}(0.0)$ \\
\hline DER067 & $700.2^{c}(80.9)$ & $4.4^{\mathrm{b}}(0.5)$ & $12.8^{\mathrm{bc}}(1.5)$ & $\operatorname{tr}$ & $0.3^{\mathrm{a}}(0.0)$ & $1.2^{\mathrm{b}}(0.1)$ & $3.6^{\mathrm{b}}(0.4)$ & $4.3^{\mathrm{a}}(0.5)$ & $0.6^{\mathrm{a}}(0.1)$ & $42.4^{\mathrm{ab}}(4.9)$ & $58.7^{\mathrm{d}-\mathrm{g}}(6.8)$ & $6.4^{\mathrm{b}-\mathrm{f}}(0.7)$ & $29.8^{\mathrm{abc}}(3.4)$ & $0.1^{\mathrm{b}}(0.0)$ & $0.2^{\mathrm{a}}(0.0)$ & $0.1^{\mathrm{ab}}(0.0)$ \\
\hline DER068 & $223.8^{\mathrm{b}}(43.9)$ & $1.4^{\mathrm{b}}(0.3)$ & $3.9^{\mathrm{b}}(0.8)$ & - & $0.5^{\mathrm{a}}(0.1)$ & $0.3^{\mathrm{a}}(0.1)$ & $0.8^{\mathrm{a}}(0.2)$ & $21.4^{a}(4.2)$ & $1.1^{\mathrm{a}}(0.2)$ & $88.5^{\mathrm{b}}(17.4)$ & $117.6^{\mathrm{fg}}(23.1)$ & $19.7^{f}(3.9)$ & $27.4^{\mathrm{abc}}(5.4)$ & $0.4^{\mathrm{b}}(0.1)$ & $2.7^{b}(0.5)$ & $0.2^{b}(0.0)$ \\
\hline DER069 & $483.3^{\mathrm{bc}}(72.6)$ & $4.1^{\mathrm{b}}(0.6)$ & $9.6^{\text {bc }}(1.4)$ & $\operatorname{tr}$ & $0.8^{\mathrm{a}}(0.1)$ & $1.6^{\mathrm{b}}(0.2)$ & $1.5^{\mathrm{b}}(0.2)$ & $22.1^{\mathrm{a}}(3.3)$ & $0.9^{\mathrm{a}}(0.1)$ & $65.5^{\mathrm{ab}}(9.8)$ & $46.0^{\mathrm{d}-\mathrm{g}}(6.9)$ & $4.8^{\mathrm{a}-\mathrm{e}}(0.7)$ & $20.9^{\mathrm{ab}}(3.1)$ & $0.1^{\mathrm{b}}(0.0)$ & $4.1^{\mathrm{b}}(0.6)$ & $0.1^{\mathrm{a}}(0.0)$ \\
\hline DER071 & $470.4^{\mathrm{bc}}(77.7)$ & $7.8^{\mathrm{b}}(1.3)$ & $16.8^{\mathrm{bc}}(2.8)$ & $\operatorname{tr}$ & $0.7^{\mathrm{a}}(0.1)$ & $2.3^{\mathrm{b}}(0.4)$ & $0.6^{\mathrm{b}}(0.1)$ & $3.4^{\mathrm{a}}(0.6)$ & $0.2^{\mathrm{a}}(0.0)$ & $4.2^{\mathrm{a}}(0.7)$ & $46.5^{\mathrm{c}-\mathrm{g}}(7.7)$ & $6.0^{\mathrm{b}-\mathrm{e}}(1.0)$ & $43.8^{\mathrm{abc}}(7.2)$ & $0.4^{\mathrm{b}}(0.1)$ & $2.2^{\mathrm{b}}(0.4)$ & $0.5^{\mathrm{b}}(0.1)$ \\
\hline DER082 & $630.8^{\mathrm{c}}(79.6)$ & $4.1^{\mathrm{b}}(0.5)$ & $8.8^{\mathrm{bc}}(1.1)$ & $\operatorname{tr}$ & $0.5^{\mathrm{a}}(0.1)$ & $1.2^{\mathrm{b}}(0.1)$ & $2.0^{\mathrm{b}}(0.3)$ & $12.9^{\mathrm{a}}(1.6)$ & $0.8^{\mathrm{a}}(0.1)$ & $81.5^{\mathrm{b}}(10.3)$ & $23.7^{\mathrm{b}-\mathrm{d}}(3.0)$ & $3.2^{\mathrm{a}-\mathrm{c}}(0.4)$ & $20.4^{\mathrm{abc}}(2.6)$ & $0.1^{\mathrm{b}}(0.0)$ & $2.4^{\mathrm{b}}(0.3)$ & $0.0^{\mathrm{a}}(0.0)$ \\
\hline DER087 & $866.3^{\mathrm{c}}(81.2)$ & $11.7^{\mathrm{b}}(1.1)$ & $20.8^{c}(2.0)$ & $\operatorname{tr}$ & $0.5^{\mathrm{a}}(0.0)$ & $8.2^{\mathrm{b}}(0.8)$ & $1.4^{\mathrm{b}}(0.1)$ & $5.8^{\mathrm{a}}(0.5)$ & $0.5^{\mathrm{a}}(0.0)$ & $11.9^{\mathrm{ab}}(1.1)$ & $37.6^{\mathrm{c}-\mathrm{f}}(3.5)$ & $6.4^{\mathrm{b}-\mathrm{e}}(0.6)$ & $90.4^{\mathrm{bc}}(8.5)$ & $0.4^{\mathrm{b}}(0.0)$ & $3.0^{\mathrm{b}}(0.3)$ & $2.3^{\mathrm{b}}(0.2)$ \\
\hline DER089 & $1069.8^{\mathrm{c}}(88.8)$ & $10.5^{\mathrm{b}}(0.9)$ & $10.5^{\mathrm{bc}}(0.9)$ & $\operatorname{tr}$ & $0.5^{\mathrm{a}}(0.0)$ & $3.7^{\mathrm{b}}(0.3)$ & $2.7^{\mathrm{b}}(0.2)$ & $5.4^{\mathrm{a}}(0.4)$ & $1.1^{\mathrm{a}}(0.1)$ & $42.0^{\mathrm{ab}}(3.5)$ & $8.9^{\mathrm{ab}}(0.7)$ & $1.8^{\mathrm{a}}(0.1)$ & $462^{\mathrm{abc}}(3.8)$ & $0.1^{\mathrm{b}}(0.0)$ & $1.5^{\mathrm{b}}(0.1)$ & $0.8^{\mathrm{b}}(0.1)$ \\
\hline DER096 & $668.9^{c}(78.2)$ & $9.0^{\mathrm{b}}(1.1)$ & $15.1^{\mathrm{bc}}(1.8)$ & $\operatorname{tr}$ & $0.4^{\mathrm{a}}(0.0)$ & $2.3^{\mathrm{b}}(0.3)$ & $1.1^{\mathrm{b}}(0.1)$ & $11.1^{\mathrm{a}}(1.3)$ & $0.9^{\mathrm{a}}(0.1)$ & $37.0^{\mathrm{ab}}(4.3)$ & $28.2^{\mathrm{b}-\mathrm{d}}(3.3)$ & $7.4^{\mathrm{b}-\mathrm{f}}(0.9)$ & $72.5^{\mathrm{bc}}(8.5)$ & $0.2^{\mathrm{a}}(0.0)$ & $0.8^{\mathrm{ab}}(0.1)$ & $0.5^{\mathrm{b}}(0.1)$ \\
\hline DER102 & $646.5^{\mathrm{c}}(69.5)$ & $7.9^{\mathrm{b}}(0.8)$ & $15.2^{\mathrm{bc}}(1.6)$ & $\operatorname{tr}$ & $0.5^{\mathrm{a}}(0.1)$ & $2.7^{\mathrm{b}}(0.3)$ & $1.5^{\mathrm{b}}(0.2)$ & $5.3^{\mathrm{a}}(0.6)$ & $0.8^{\mathrm{a}}(0.1)$ & $41.6^{\mathrm{ab}}(4.5)$ & $83.1^{\mathrm{d}-\mathrm{g}}(8.9)$ & $7.3^{\mathrm{b}-\mathrm{f}}(0.8)$ & $112.6^{\mathrm{c}}(12.1)$ & $0.3^{\mathrm{b}}(0.0)$ & $4.1^{\mathrm{b}}(0.4)$ & $0.4^{\mathrm{b}}(0.0)$ \\
\hline DER103 & $918.7^{\mathrm{C}}(89.5)$ & $7.9^{\mathrm{b}}(0.8)$ & $8.5^{\mathrm{bc}}(0.8)$ & $\operatorname{tr}$ & $0.2^{\mathrm{a}}(0.0)$ & $1.6^{\mathrm{b}}(0.2)$ & $1.1^{\mathrm{b}}(0.1)$ & $4.4^{\mathrm{a}}(0.4)$ & $0.7^{\mathrm{a}}(0.1)$ & $36.9^{\mathrm{ab}}(3.6)$ & $22.0^{\mathrm{b}-\mathrm{d}}(2.1)$ & $2.4^{\mathrm{ab}}(0.2)$ & $21.0^{\mathrm{abc}}(2.0)$ & $0.1^{\mathrm{b}}(0.0)$ & $0.3^{\mathrm{a}}(0.0)$ & $0.2^{\mathrm{b}}(0.0)$ \\
\hline Mean & 680.6 & 6.6 & 11.6 & $t r$ & 0.5 & 2.9 & 1.5 & 10.3 & 0.9 & 46.7 & 51.4 & 7.0 & 56.4 & 0.2 & 2.3 & 0.6 \\
\hline
\end{tabular}

Different letters within columns for the GC-peak area units indicate significant differences according to the Student-Newman-Keuls test $(p=0.05)$. tr traces; - not detected. For compounds identities refer to Table 3 . 


\subsubsection{Group of Isothiocyanates}

Overall, seven different ITCs were identified in the present work among the evaluated wall rocket materials (Table 3). Allyl ITC (I1) was reported as the main sulfurous compound in this chemical group and was also the main VOC in the total profile of the species (Table 4). Additionally, other ITCS were identified and included 3-butenyl ITC (I2), 3-methylbutyl ITC (I3) and benzyl ITC (I6), together with hexyl ITC (I5), phenylethyl ITC (I7) and pentyl ITC (I4), which often showed the lowest levels (Table 4).

In agreement with our results, these compounds have been previously described in wall rocket microgreens and seedlings [4]. By contrast, for mature leaves, stems and pods, Clemente-Villalba et al. [33] only identified allyl ITC, 3-butenyl ITC and phenylethyl ITC. The presence and relative contribution of individual ITCs in wall rocket differs considerably to those reported in the rocket crops $[11,22,26,29,34-40]$. Thus, three compounds identified in the present study for wall rocket (i.e., allyl ITC, benzyl ITC and phenylethyl ITC) were not reported in the literature for the related rocket crops (Table S1). Similarly, other sulfur-containing compounds described in both salad and wild rocket were not found in our wall rocket materials. Since individual ITCs have been described to add specific sensory descriptors [13], differences in the ITCs fraction (both qualitative and relative abundances) can result in remarkable differences in flavor. In this context, we hypothesize that wall rocket should be marketed as a new crop, as its aroma is not greatly correlated to rocket crops [4]. In fact, previous works evaluating the sensory attributes and consumers acceptance suggest that comparing wall rocket with the commercial rocket crops may increase consumers rejection [3] as the species would show an unexpected, no rocket-like flavor.

On the contrary, the volatile profile of wall rocket could be more related to other Brassicaceae crops such as wasabi, mustard or horseradish. For all these crops, allyl ITC has been described as the predominant ITC [4,41-43]. Providing characteristic flavor notes that are described as pungent, sulfurous, bitter, lachrymose and mustard-, onion- and wasabi-like [13,42], this compound would be greatly responsible of the common pungent notes that all these crops show. In addition, 3-butenyl ITC, 3-methylbutyl ITC and phenylethyl ITC have been also described in the volatile profile of horseradish and mustard, the former crop highlighting for the content in phenylethyl ITC as second predominant ITC $[4,42]$. By contrast, phenylethyl ITC has not been reported in wasabi tissues $[44,45]$ although it can be found in wasabi-based products [4].

Regarding the comparison of the ITCs fraction in the populations of wall rocket, it was found that most materials showed similar profiles in qualitative terms but some quantitative differences were established (Table 4). As main compound, allyl ITC showed an average relative abundance of $77 \%$, which raised above $88 \%$ in populations DER089 and DER103. From them, DER089 showed the highest absolute value (GC-peak area units). There was a 6-fold difference between this population and the population with the lowest total value (DER045, $183 \times 10^{6}$ ), which also showed exceptionally low levels in relative abundance (45\%). In addition, 3-butenyl ITC (I2) and 3-methylbutyl ITC (I3) added an average relative abundance of $2.0 \%$ (Table 4). In both cases, DER045 showed the lowest absolute level and DER087, the highest one. The differences in GC-peak area units among them were 7-fold and 11-fold for compounds I2 and I3, respectively. Moreover, benzyl ITC (I6) and phenylethyl ITC (I7) were detected in all materials except for DER045, and hexyl ITC (I5) was only detected in this material as traces. Finally, pentyl ITC (I4) was only identified as traces (18 populations) or not detected (Table 4).

Considering that both the concentration and the relative abundance of individual ITCs in a food matrix influence the consumers perception $[13,46]$, differences among populations may be exploited for developing commercial varieties of different aroma and pungency. In particular, our results suggest that populations like DER089 and DER103 could be selected for obtaining commercial varieties of high pungency. On the contrary, the lower levels found in DER045 and DER068 suggest that these populations could show lower pungency perception. 


\subsubsection{Group of Esters and Other Compounds}

Esters represented the second group of importance in the profile of wall rocket and was represented by five compounds (Table 3). Despite all populations showed similar levels for this group, significant differences were found for individual compounds (Table 4). Two esters, identified as cis-3-hexenyl isovalerate (E4) and cis-3-hexenyl butyrate (E3), contributed to approximately $11 \%$ of the total abundance (Table 4). Differences in absolute levels were found among populations for both compounds, reaching almost 20-fold. Thus, DER045 and DER040 showed the highest absolute levels for cis-3-hexenyl isovalerate (E4) and cis-3-hexenyl butyrate (E3), respectively, while the lowest values were found in DER057 and DER071, respectively. Hexyl-butyrate (E2) was the compound in this fraction showing the lowest levels. Cis-3-hexenyl propionate (E1) and cis-3-hexenyl valerate (E5) were found at intermediate levels. From them, cis-3-hexenyl propionate (E1) showed a high intra-population variation that reduced differences among materials. On the contrary, cis-3-hexenyl valerate (E5) was mainly affected by the accession and therefore significant differences were found. Thus, DER057 and DER089 showed the lowest levels, while it was found at the highest levels in DER068 (Table 4).

On the other hand, the miscellaneous group was mainly represented by cis-3-hexen-1-ol (O1). This alcohol accounted on average for $6.4 \%$ of the total volatile profile. DER045 showed the lowest value in absolute terms, which was almost 4-fold times lower than in DER102 (Table 4).

Both the alcohol cis-3-hexen-1-ol and the esters found in the present study are known as green leaf volatiles (GLV). This class of compounds is commonly released from leafy vegetables, flowers and fruits following damage $[47,48]$, for example due to herbivore attack but also when cutting during food preparation. The process is suggested to be related to the defense system of plants [25], but it is also of interest for sensorial studies and consumers acceptance as the compounds released provide a characteristic green leaf odor [47]. In agreement with our results, cis-3-hexen-1-ol has been previously found as the main alcohol in other Brassicaceae including rocket crops, radish or horseradish [29,31,34,42] and it would provide grassy, green notes [42]. This compound is commonly found together with other GLV alcohols, but especially with GLV aldehydes in leaf material [22,33], although this co-occurrence is not always so clear [31]. On the other hand, GL esters are not commonly described as headspace volatiles in rocket crops or found at low relative abundances [29,31,34], but results differ in literature. For example, Jirovetz et al. [26] also found cis-3-hexenyl butyrate as the main ester in salad rocket leaves, sharing similar levels as the alcohol cis-3-hexen-1-ol as in some of the populations of wall rocket tested in the current study. Interestingly, López-Gresa et al. [25] identified esters of cis-3-hexen-1-ol including cis-3-hexenyl- propionate, butyrate and isobutyrate as prominent VOCs in tomato leaves released as defensive mechanism. Considering that our methodology provoked chemical damage by cutting, the abundance of esters in our materials could be explained by the activation of a similar defensive response. However, this issue needs more attention and should be further studied.

Together with their involvement in the defense system, esters also provide aroma notes like green, fruity, fresh and herbal $[26,49]$. In particular, the main esters determined in the current study are related to green, fresh and herbal aroma. In this sense, DER045 and DER040 could be candidates for developing varieties with enhanced green notes. Furthermore, the population DER102 may be considered as it complemented the highest levels of the alcohol cis-3-hexen-1-ol with also high levels of the ester cis-3-hexenyl isovalerate.

\subsection{Accumulation of Sinigrin}

Together with the analysis of the volatiles profile, the accumulation of GSLs in these materials of wall rocket was also evaluated. Sinigrin was identified as the main glucosinolate in the leaves of wall rocket and was, therefore, the target compound quantified in our HPLC analysis. The identification of sinigrin as the main GSL is in agreement with the previous description of the species $[17,18]$. This aliphatic GSL is also of relevance in other Brassicaceae crops such as kale, horseradish or mustard [50-52], while its occurrence in the commercial rocket crops has not been described so far $[8,17,53,54]$. 
The average value for the total of populations was $7.76 \mathrm{mg} 100 \mathrm{~g}^{-1} \mathrm{FW}$, while differences among

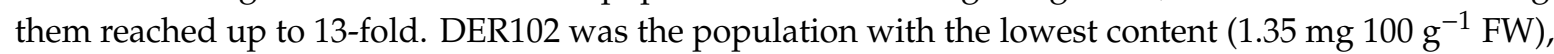
while DER064 showed the highest value (18.18 mg $100 \mathrm{~g}^{-1} \mathrm{FW}$ ) (Table 5). Nevertheless, and despite the broad range found among populations, the contents were significantly lower than reported by other authors, between 8.4 and $13.4 \mathrm{mg} \mathrm{g}^{-1} \mathrm{DW}[17,18]$. There are several factors that can affect the accumulation of glucosinolates in plant tissues including the growing conditions [55], and this may explain in part the differences found among the current study and previous reports. Thus, modifying culture practices may increase the levels of sinigrin as main GSL of wall rocket. In fact, we found that some populations used in the present study showed up to 15-fold increase when they were tested under field conditions [8]. Therefore, our results suggest that the conditions used in the present work could be adapted to increase the accumulation of sinigrin.

Table 5. Content in sinigrin (mg $\left.100 \mathrm{~g}^{-1} \mathrm{FW}\right)$ and coefficient of variation $(\mathrm{CV}, \%)$ in the 24 populations of wall rocket evaluated $(n=3)$.

\begin{tabular}{lll}
\hline & Sinigrin & CV (\%) \\
\hline DER006 & $6.60^{\mathrm{de}}$ & 1.7 \\
DER008 & $8.07^{\mathrm{ef}}$ & 7.3 \\
DER014 & $9.52^{\mathrm{f}}$ & 10.2 \\
DER021 & $3.31^{\mathrm{c}}$ & 4.9 \\
DER039 & $10.13^{\mathrm{f}}$ & 27.6 \\
DER040 & $5.97^{\mathrm{cd}}$ & 7.3 \\
DER045 & $5.82^{\mathrm{cd}}$ & 6.6 \\
DER052 & $4.65^{\mathrm{c}}$ & 18.0 \\
DER056 & $6.88^{\mathrm{de}}$ & 13.3 \\
DER057 & $9.04^{\mathrm{f}}$ & 10.1 \\
DER060 & $12.12^{\mathrm{g}}$ & 6.5 \\
DER061 & $16.87^{\mathrm{h}}$ & 1.1 \\
DER062 & $5.79^{\mathrm{cd}}$ & 11.4 \\
DER064 & $18.18^{\mathrm{h}}$ & 4.5 \\
DER067 & $2.28^{\mathrm{b}}$ & 24.8 \\
DER068 & $3.06^{\mathrm{c}}$ & 11.5 \\
DER069 & $6.04^{\mathrm{cd}}$ & 14.4 \\
DER071 & $15.79^{\mathrm{h}}$ & 6.4 \\
DER082 & $3.39^{\mathrm{c}}$ & 10.3 \\
DER087 & $15.48^{\mathrm{h}}$ & 15.5 \\
DER089 & $3.60^{\mathrm{c}}$ & 7.6 \\
DER096 & $3.26^{\mathrm{c}}$ & 16.7 \\
DER102 & $1.35^{\mathrm{a}}$ & 8.1 \\
DER103 & $8.98^{\mathrm{f}}$ & 8.3 \\
Mean & 7.76 & 62.5 \\
\hline
\end{tabular}

Different letters indicate significant differences according to the Student-Newman-Keuls test $(p=0.05)$.

Together with sinigrin, the identification of other ITCs in the volatile profile could indicate the co-occurrence of other minor GSLs in wall rocket, although it could not be detected in our chromatograms. Therefore, we considered clarifying this issue by performing an additional analysis with an equipment that could provide greater accuracy. Thus, two additional samples, which were grown as described in Guijarro-Real et al. [10] to increase levels of GSLs, were analyzed by the external service Analysis of Quality in Plants from the Misión Biológica de Galicia-Consejo Superior de Investigaciones Científicas (MBG-CSIC) with ultra-high performance liquid chromatography (UHPLC). A total of six GSLs were identified in this analysis: glucoiberin, sinigrin, glucotropaeolin, glucobrassicin, neoglucobrassicin, and a sixth compound that may correspond to gluconasturtiin/methoxyglucobrassicin -the peak could not be clearly identified at the low levels in which it was found, and may correspond to gluconasturtiin, methoxyglucobrassicin or a mixture of both- (Figure 1). Glucotropaeolin and gluconasturtiin derive in benzyl ITC and phenylethyl ITC, 
respectively [13], both detected in the ITCs fraction. By contrast, the breakdown product of glucoiberin could not be identified within the VOCs. Finally, indolic GSLs (glucobrassicin, methoxyglucobrassicin and neoglucobrassicin) derive in unstable ITCs that spontaneously decompose to other products [56], so the corresponding ITCs would not be easily identified. Differences between the GSLs and ITCs fractions represent an interrogative that should be clarified in new studies, for which we suggest the use of concentration steps during the extraction process with an attempt to increase the chromatogram signals of the target compounds.

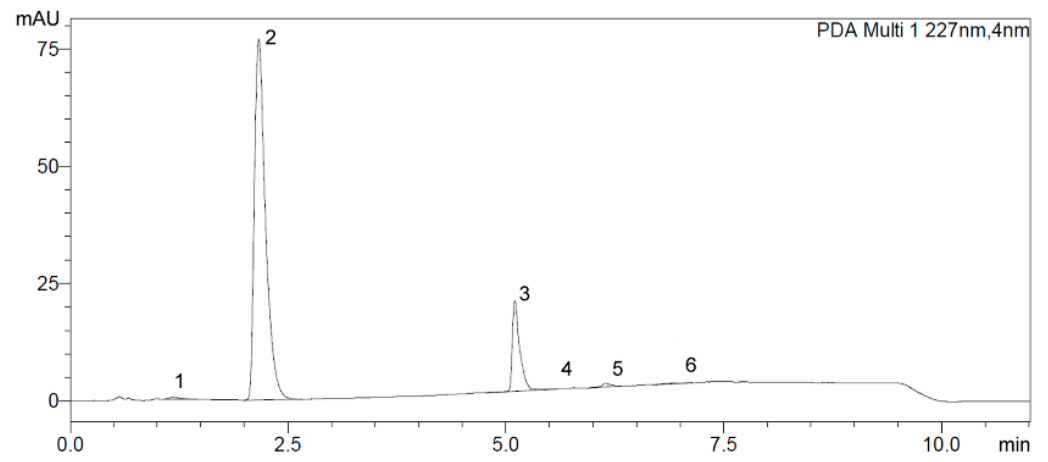

Figure 1. Chromatogram of the GLSs profile in wall rocket obtained with UHPLC by the external service. 1: glucoiberin; 2: sinigrin; 3: glucotropaeolin; 4: glucobrassicin; 5: gluconasturtiin/methoxyglcucobrassicin; 6: neoglucobrassicin.

\subsection{Principal Component Analysis and Hierarchical Cluster Analysis}

A Principal Component Analysis was performed using the mean GC-peak area values of individual VOCs, total levels, and the content in sinigrin for each population (Figure 2). The first two components explained together $61.1 \%$ of the total variance, with the first component contributing to $43.1 \%$ of variation. The first component was positively correlated to the ITCs compounds, where values for component loadings were $>0.2$ (Figure 2a). Allyl ITC (I1) grouped together with the total level of ITCs and the total level of VOCs. This result is in agreement with the previous identification of allyl ITC as the main volatile in wall rocket and therefore the greatest contributor to the total added area of VOCs. The first component also had a positive correlation with the content in sinigrin. On the contrary, esters were not grouped by neither the first nor the second component. As exception, cis-3-hexenyl isovalerate (E4) and cis-3-hexenyl valerate (E5) clustered together; indeed, the Spearman ordinal correlation between both compounds was highly significant and positive $(\rho=0.945 ; p<0.001)$. On the other hand, the second component accounted for $18.0 \%$ of total variance (Figure 2a). It was also positively correlated to the content in sinigrin. In addition, this component clustered three of the four compounds included in the miscellaneous group. Cis-3-hexen-1-ol (O1) was closely grouped with the total sum of this group as it was the greatest contributor.

The relationship between individual compounds was also evaluated by means of the Hierarchical Cluster Analysis (Figure 3). Two main clusters were established, C1 and C2, and were powerful in grouping together the esters (cluster C1) and, separately, the ITCs (cluster C2). The compounds of the miscellaneous group were included in the previous groups as described: decanal $(\mathrm{O} 2)$ and tetradecane (O3) within the cluster $\mathrm{C} 1$, and cis-3-hexen-1-ol (O1) and $\beta$-ionone (O4) as part of the cluster $\mathrm{C} 2$. Within the cluster $\mathrm{C} 1$, the compounds cis-3-hexenyl isovalerate (E4) and cis-3-hexenyl valerate (E5) clustered together, as it was observed in the PCA loading plot (Figure 2a, Figure 3). Regarding the cluster C2, here it was included the GSL sinigrin, which mainly grouped with allyl ITC (I1), hexyl ITC (I5), benzyl ITC (I6) and phenylethyl ITC (I7) (Figure 3). 


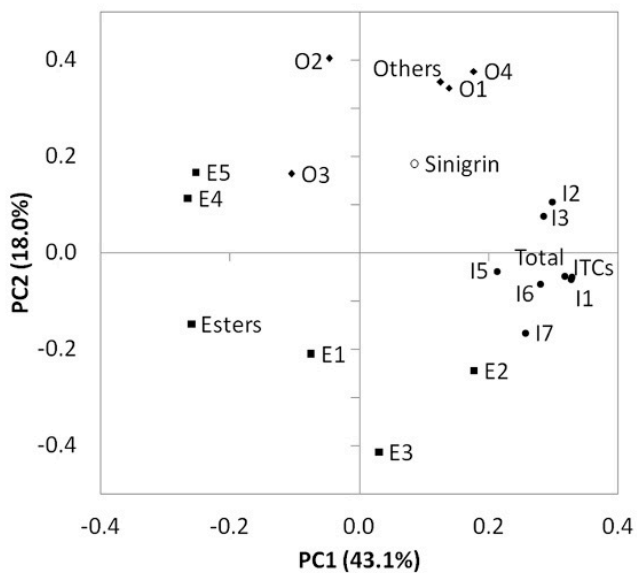

(a)

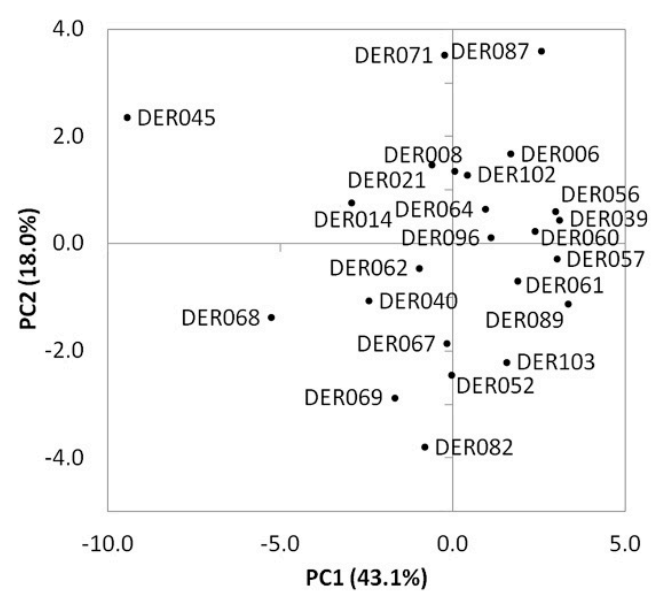

(b)

Figure 2. Principal Component Analysis of the 24 populations of wall rocket evaluated in the study, in the first (PC1) and second (PC2) components. (a) PCA loading plot for the volatile organic compounds and content in sinigrin. (b) PCA score plot for the populations. Data were averaged for each accession. For compound identities refer to Table 3.

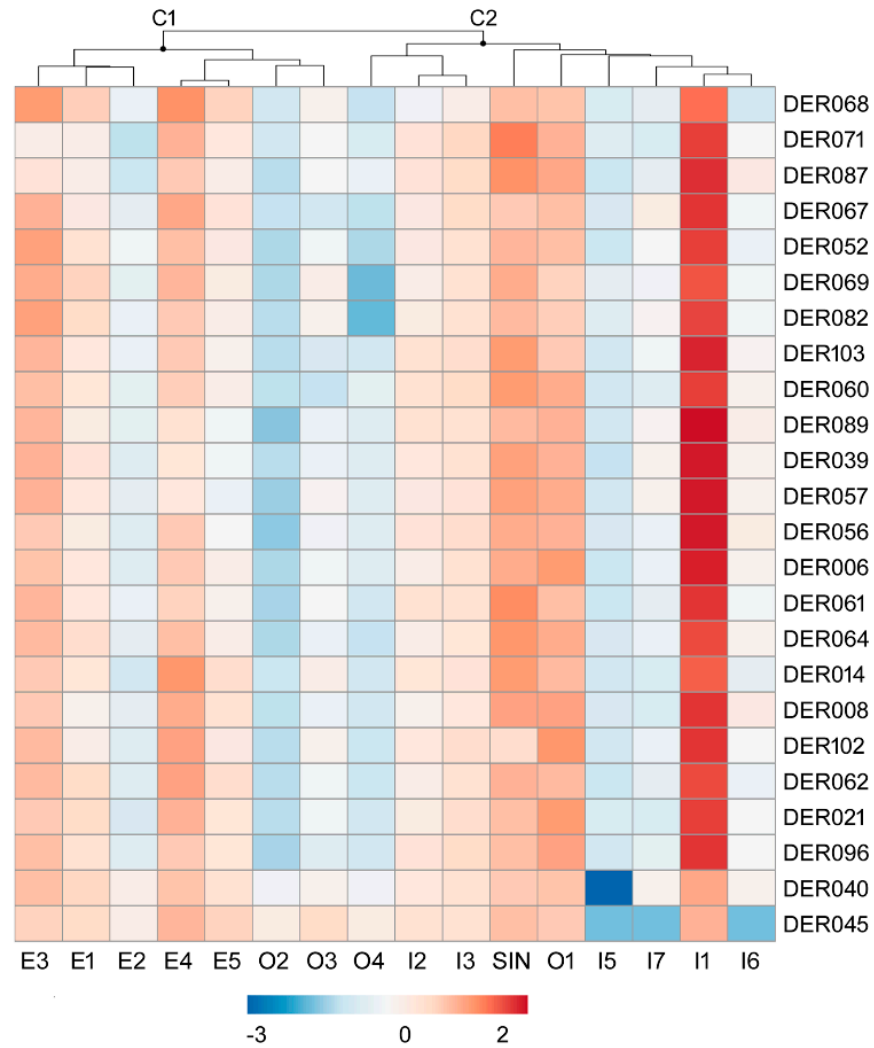

Figure 3. Hierarchical Cluster Analysis for the identified volatile compounds and content in sinigrin in the 24 populations of wall rocket. Data represent the average value for each population $(n=3)$. For compound identities refer to Table 3.

On the other hand, the score plot of the PCA revealed the relationship between the materials (Figure 2b). Most of the populations grouped close in the analysis, although some populations were separated along either the first or second component. Thus, DER045 clearly separated in the first component, placed on the left of the graphic. As previously described, this population had the lowest level in ITCs combined with high levels of esters (Table 2). Its different profile was also observed in 
the Hierarchical Cluster Analysis (Figure 3). Similarly, DER068 was also separated within this first component (Figure $2 b$ ). This population showed the highest level of esters, but also an increase in the level of ITCs compared to DER045 (Table 2). Regarding the second component, it grouped populations DER071 and DER087 in a separated cluster on the top of the graphic (Figure 2b). Both populations shared a high accumulation of sinigrin and low in esters (Table 2, Table 5).

The combined results of the PCA and Hierarchical Cluster Analysis provided information of interest for future breeding programs. The accumulation of GSL-derived compounds is determinant for the bitterness and pungency of the Brassicaceae, due to the fact that many of these compounds provide characteristic flavors like hot, pungent, mustard-like or cabbage-like to the plant materials in which they are found [13]. The perception of such pungency can be modified due to the increase/decrease of the levels in which the individual ITCs are found. However, there are other factors that would affect this perception as well. Thus, the presence and relative abundance of other families of volatiles like the green leaf volatiles can reduce the pungent feeling, as the results of Bell et al. [57] suggest. In this sense, the present work identified the population DER045, which combined reduced levels of ITCs and high levels of esters, as a possible candidate in breeding programs aimed at obtaining a variety of mild pungency and a more notable green flavor. Furthermore, population DER068 could be also tested in these terms, as its profile suggests that it could be also mild pungent according to the levels of esters but accumulate at the same time higher levels of ITCs with the potential health benefits associated. Thus, both accessions could be initially selected for further studies including sniffing tests, sensory perception analyses and consumers acceptance. By contrast, several populations that clustered together could be selected for obtaining commercial varieties with enhanced pungent flavor. Since their volatile profiles did not differ greatly, here we suggest that other traits such as the morphology and growth traits could be also considered for the breeding programs [9].

\section{Conclusions}

The volatiles profile of wall rocket baby-leaves was rich in ITCs, mainly allyl ITC, which would be the major factor providing the pungent flavor of this species. The ITCs fraction differed considerably from the one reported in rocket crops, which could result in a market opportunity. In addition, esters were of high relevance in the profile, mainly cis-3-hexenyl butyrate and cis-3-hexenyl isovalerate. DER045 was identified as a population with a profile that was significantly different from the rest. Due to the low level of ITCs and high relative abundance of esters, this population could be selected for the development of a mild-pungent variety.

In addition, sinigrin was identified as the main GSL in the wall rocket materials. The accumulation among populations showed a broad range. An additional analysis showed that other GSLs would be present in this species as well, as it was suggested by the profile in volatiles. Overall, the work increases the knowledge in the volatiles profile of wall rocket, providing also relevant information in the variation for sinigrin and the presence of novel GSLs. The results obtained are useful for breeding programs aimed to improve the nutritional quality and pungency of wall rocket as a new crop.

Supplementary Materials: The following are available online at http://www.mdpi.com/2073-4395/10/6/802/s1, Table S1: Isothiocyanates fraction described in wall rocket and qualitative comparison with the related wild and salad rocket crops according to the literature.

Author Contributions: Conceptualization, A.R.-B. and A.F.; Data curation, C.G.-R.; Formal analysis, C.G.-R. and A.F.; Investigation, C.G.-R.; Methodology, C.G.-R., A.R.-B. and A.F.; Project administration, A.R.-B. and A.F.; Resources, A.R.-B. and A.F.; Software, C.G.-R.; Supervision, A.R.-B. and A.F.; Validation, C.G.-R.; Visualization, C.G.-R., A.R.-B. and A.F.; writing-original draft preparation, C.G.-R., A.R.-B. and A.F.; writing-review and editing, C.G.-R., A.R.-B. and A.F. All authors have read and agreed to the published version of the manuscript.

Funding: This research received no external funding.

Acknowledgments: C.G. thanks the Ministerio de Educación, Cultura y Deporte of Spain (MECD) for the financial support by means of a predoctoral FPU grant (FPU14-06798).

Conflicts of Interest: The authors declare no conflict of interest. 


\section{References}

1. Tripodi, P.; Francese, G.; Mennella, G. Rocket salad: Crop description, bioactive compounds and breeding perspectives. Adv. Hortic. Sci. 2017, 31, 107-113. [CrossRef]

2. Guijarro-Real, C.; Navarro, A.; Esposito, S.; Festa, G.; Macellaro, R.; Di Cesare, C.; Fita, A.; Rodríguez-Burruezo, A.; Cardi, T.; Prohens, J.; et al. Large scale phenotyping and molecular analysis in a germplasm collection of rocket salad (Eruca vesicaria) reveal a differentiation of the gene pool by geographical origin. Euphytica 2020, 216, 1-20. [CrossRef]

3. D'Antuono, F.; Elementi, S.; Neri, R. Exploring new potential health-promoting vegetables: Glucosinolates and sensory attributes of rocket salads and related Diplotaxis and Eruca species. J. Sci. Food Agric. 2009, 89, 713-722. [CrossRef]

4. Guijarro-Real, C.; Prohens, J.; Rodríguez-Burruezo, A.; Fita, A. Consumers acceptance and volatile profile of wall rocket (Diplotaxis erucoides). Food Res. Int. 2020, 132, 109008. [CrossRef]

5. Pignone, D.; Martínez-Laborde, J.B. Diplotaxis. In Wild Crop Relatives: Genomic and Breeding Resources. Oilseeds; Kole, C., Ed.; Springer: Berlin, Germany, 2011; pp. 137-147.

6. Couplan, F. Le Régal Végétal. Reconnaître et Cuisiner les Plantes Comestibles; Sang de la Terre: Paris, France, 2015.

7. Guarrera, P.; Savo, V. Wild food plants used in traditional vegetable mixtures in Italy. J. Ethnopharmacol. 2016, 185, 202-234. [CrossRef]

8. Guijarro-Real, C.; Adalid-Martínez, A.M.; Aguirre, K.; Prohens, J.; Rodríguez-Burruezo, A.; Fita, A. Growing Conditions Affect the Phytochemical Composition of Edible Wall Rocket (Diplotaxis erucoides). Agronomy 2019, 9, 858. [CrossRef]

9. Guijarro-Real, C.; Prohens, J.; Rodríguez-Burruezo, A.; Fita, A. Morphological Diversity and Bioactive Compounds in Wall Rocket (Diplotaxis erucoides (L.) DC.). Agronomy 2020, 10, 306. [CrossRef]

10. Guijarro-Real, C.; Prohens, J.; Rodríguez-Burruezo, A.; Fita, A. Potential of wall rocket (Diplotaxis erucoides) as a new crop: Influence of the growing conditions on the visual quality of the final product. Sci. Hortic. 2019, 258, 108778. [CrossRef]

11. Bell, L.; Yahya, H.N.; Oloyede, O.O.; Methven, L.; Wagstaff, C. Changes in rocket salad phytochemicals within the commercial supply chain: Glucosinolates, isothiocyanates, amino acids and bacterial load increase significantly after processing. Food Chem. 2017, 221, 521-534. [CrossRef]

12. Baenas, N.; Cartea, M.E.; Moreno, D.A.; Tortosa, M.; Francisco, M. Processing and cooking effects on glucosinolates and their derivatives. In Glucosinolates: Properties, Recovery, and Applications; Galanakis, C.M., Ed.; Elsevier Inc., Academic Press: Cambridge, MA, USA, 2020; pp. 181-212.

13. Bell, L.; Oloyede, O.O.; Lignou, S.; Wagstaff, C.; Methven, L. Taste and Flavor Perceptions of Glucosinolates, Isothiocyanates, and Related Compounds. Mol. Nutr. Food Res. 2018, 62, 1700990. [CrossRef]

14. Sávio, A.L.V.; Da Silva, G.N.; Salvadori, D.M.F. Inhibition of bladder cancer cell proliferation by allyl isothiocyanate (mustard essential oil). Mutat. Res. Mol. Mech. Mutagen. 2015, 771, 29-35. [CrossRef] [PubMed]

15. Sávio, A.L.V.; Da Silva, G.N.; De Camargo, E.A.; Salvadori, D.M.F. Cell cycle kinetics, apoptosis rates, DNA damage and TP53 gene expression in bladder cancer cells treated with allyl isothiocyanate (mustard essential oil). Mutat. Res. Mol. Mech. Mutagen. 2014, 762, 40-46. [CrossRef] [PubMed]

16. Rajakumar, T.; Pugalendhi, P.; Thilagavathi, S. Dose response chemopreventive potential of allyl isothiocyanate against 7,12-dimethylbenz(a)anthracene induced mammary carcinogenesis in female Sprague-Dawley rats. Chem. Interact. 2015, 231, 35-43. [CrossRef] [PubMed]

17. Di Gioia, F.; Avato, P.; Serio, F.; Argentieri, M. Glucosinolate profile of Eruca sativa, Diplotaxis tenuifolia and Diplotaxis erucoides grown in soil and soilless systems. J. Food Compos. Anal. 2018, 69, 197-204. [CrossRef]

18. D'Antuono, F.; Elementi, S.; Neri, R. Glucosinolates in Diplotaxis and Eruca leaves: Diversity, taxonomic relations and applied aspects. Phytochemistry 2008, 69, 187-199. [CrossRef] [PubMed]

19. Guijarro-Real, C.; Adalid-Martínez, A.M.; Gregori-Montaner, A.; Prohens, J.; Rodríguez-Burruezo, A.; Fita, A. Factors affecting germination of Diplotaxis erucoides and their effect on selected quality properties of the germinated products. Sci. Hortic. 2020, 261, 109013. [CrossRef]

20. Guijarro-Real, C.; Rodríguez-Burruezo, A.; Prohens, J.; Raigón, M.D.; Fita, A. HS-SPME analysis of the volatiles profile of water celery (Apium nodiflorum), a wild vegetable with increasing culinary interest. Food Res. Int. 2018, 121, 765-775. [CrossRef] 
21. Moreno, E.; Fita, A.; González-Mas, M.C.; Rodríguez-Burruezo, A. HS-SPME study of the volatile fraction of Capsicum accessions and hybrids in different parts of the fruit. Sci. Hortic. 2012, 135, 87-97. [CrossRef]

22. Bell, L.; Spadafora, N.D.; Müller, C.T.; Wagstaff, C.; Rogers, H.J. Use of TD-GC-TOF-MS to assess volatile composition during post-harvest storage in seven accessions of rocket salad (Eruca sativa). Food Chem. 2016, 194, 626-636. [CrossRef]

23. Pasini, F.; Verardo, V.; Caboni, M.F.; D’Antuono, L.F. Determination of glucosinolates and phenolic compounds in rocket salad by HPLC-DAD-MS: Evaluation of Eruca sativa Mill. and Diplotaxis tenuifolia L. genetic resources. Food Chem. 2012, 133, 1025-1033. [CrossRef]

24. Metsalu, T.; Vilo, J. ClustVis: A web tool for visualizing clustering of multivariate data using Principal Component Analysis and heatmap. Nucleic Acids Res. 2015, 43, W566-W570. [CrossRef] [PubMed]

25. López-Gresa, M.P.; Lisón, P.; Campos, L.; Rodrigo, I.; Rambla, J.; Granell, A.; Conejero, V.; Bellés, J.M. A Non-targeted Metabolomics Approach Unravels the VOCs Associated with the Tomato Immune Response against Pseudomonas syringae. Front. Plant Sci. 2017, 8, 1188. [CrossRef]

26. Jirovetz, L.; Smith, D.; Buchbauer, G. Aroma Compound Analysis of Eruca sativa (Brassicaceae) SPME Headspace Leaf Samples Using GC, GC-MS, and Olfactometry. J. Agric. Food Chem. 2002, 50, 4643-4646. [CrossRef] [PubMed]

27. González-Mas, M.C.; Rambla, J.; Alamar, M.C.; Gutierrez, A.; Granell, A. Comparative Analysis of the Volatile Fraction of Fruit Juice from Different Citrus Species. PLoS ONE 2011, 6, e22016. [CrossRef] [PubMed]

28. Rodríguez-Burruezo, A.; Kollmannsberger, H.; González-MasM, C.; Nitz, S.; Fernando, N. HS-SPME Comparative Analysis of Genotypic Diversity in the Volatile Fraction and Aroma-Contributing Compounds of Capsicum Fruits from theannuum-chinense-frutescens Complex. J. Agric. Food Chem. 2010, 58, 4388-4400. [CrossRef] [PubMed]

29. Blažević, I.; Mastelić, J. Free and bound volatiles of rocket (Eruca sativa Mill.). Flavour Fragr. J. 2008, 23, 278-285. [CrossRef]

30. Hanschen, F.S.; Schreiner, M. Isothiocyanates, Nitriles, and Epithionitriles from Glucosinolates Are Affected by Genotype and Developmental Stage in Brassica oleracea Varieties. Front. Plant Sci. 2017, 8, 1095. [CrossRef]

31. Blažević, I.; Mastelic, J. Glucosinolate degradation products and other bound and free volatiles in the leaves and roots of radish (Raphanus sativus L.). Food Chem. 2009, 113, 96-102. [CrossRef]

32. Miyazawa, M.; Nishiguchi, T.; Yamafuji, C. Volatile components of the leaves of Brassica rapa L. var. perviridis Bailey. Flavour Fragr. J. 2005, 20, 158-160. [CrossRef]

33. Clemente-Villalba, J.; Ariza, D.; García-Garví, J.M.; Sánchez-Bravo, P.; Noguera-Artiaga, L.; Issa-Issa, H.; Hernández, F.; Carbonell-Barrachina, Á.A. Characterization and potential use of Diplotaxis erucoides as food ingredient for a sustainable modern cuisine and comparison with commercial mustards and wasabis. Eur. Food Res. Technol. 2020, 1-10. [CrossRef]

34. Raffo, A.; Masci, M.; Moneta, E.; Nicoli, S.; Del Pulgar, J.S.; Paoletti, F. Characterization of volatiles and identification of odor-active compounds of rocket leaves. Food Chem. 2017, 240, 1161-1170. [CrossRef]

35. Mastrandrea, L.; Amodio, M.L.; Pati, S.; Colelli, G. Effect of modified atmosphere packaging and temperature abuse on flavor related volatile compounds of rocket leaves (Diplotaxis tenuifolia L.). J. Food Sci. Technol. 2017, 54, 2433-2442. [CrossRef] [PubMed]

36. Miyazawa, M.; Maehara, T.; Kurose, K. Composition of the essential oil from the leaves of Eruca sativa. Flavour Fragr. J. 2002, 17, 187-190. [CrossRef]

37. Petretto, G.L.; Urgeghe, P.P.; Massa, D.; Melito, S. Effect of salinity (NaCl) on plant growth, nutrient content, and glucosinolate hydrolysis products trends in rocket genotypes. Plant Physiol. Biochem. 2019, 141, 30-39. [CrossRef] [PubMed]

38. Spadafora, N.D.; Amaro, A.L.; Pereira, M.J.V.; Müller, C.T.; Pintado, M.; Rogers, H.J.; Pintado, M.M. Multi-trait analysis of post-harvest storage in rocket salad (Diplotaxis tenuifolia) links sensorial, volatile and nutritional data. Food Chem. 2016, 211, 114-123. [CrossRef] [PubMed]

39. Spadafora, N.D.; Cocetta, G.; Ferrante, A.; Herbert, R.J.; Dimitrova, S.; Davoli, D.; Fernández, M.; Patterson, V.; Vozel, T.; Amarysti, C.; et al. Short-Term Post-Harvest Stress that Affects Profiles of Volatile Organic Compounds and Gene Expression in Rocket Salad during Early Post-Harvest Senescence. Plants 2019, 9, 4. [CrossRef] 
40. Villatoro, M.; Priego-Capote, F.; Álvarez-Sánchez, B.; Saha, S.; Philo, M.; Cano, S.O.; De Haro-Bailón, A.; Font, R.; Del Río-Celestino, M. An approach to the phytochemical profiling of rocket [Eruca sativa (Mill.) Thell]. J. Sci. Food Agric. 2013, 93, 3809-3819. [CrossRef]

41. Bending, G.; Lincoln, S.D. Characterisation of volatile sulphur-containing compounds produced during decomposition of Brassica juncea tissues in soil. Soil Boil. Biochem. 1999, 31, 695-703. [CrossRef]

42. Kroener, E.-M.; Buettner, A. Unravelling important odorants in horseradish (Armoracia rusticana). Food Chem. 2017, 232, 455-465. [CrossRef]

43. Sultana, T.; Porter, N.G.; Savage, G.; McNeil, D.L. Comparison of Isothiocyanate Yield from Wasabi Rhizome Tissues Grown in Soil or Water. J. Agric. Food Chem. 2003, 51, 3586-3591. [CrossRef]

44. DePree, J.A.; Howard, T.M.; Savage, G.P. Flavour and pharmaceutical properties of the volatile sulphur compounds of Wasabi (Wasabia japonica). Food Res. Int. 1998, 31, 329-337. [CrossRef]

45. Sultana, T.; Savage, G.P.; McNeil, D.L.; Porter, N.G.; Clark, B. Comparison of flavour compounds in wasabi and horseradish. J. Food Agric. Environ. 2003, 1, 117-121.

46. Pasini, F.; Verardo, V.; Cerretani, L.; Caboni, M.; D’Antuono, L.F. Rocket salad (Diplotaxis and Eruca spp.) sensory analysis and relation with glucosinolate and phenolic content. J. Sci. Food Agric. 2011, 91, 2858-2864. [CrossRef] [PubMed]

47. Ruther, J. Retention index database for identification of general green leaf volatiles in plants by coupled capillary gas chromatography-mass spectrometry. J. Chromatogr. A 2000, 890, 313-319. [CrossRef]

48. D'Auria, J.C.; Pichersky, E.; Schaub, A.; Hansel, A.; Gershenzon, J. Characterization of a BAHD acyltransferase responsible for producing the green leaf volatile (Z)-3-hexen-1-yl acetate in Arabidopsis thaliana. Plant J. 2006, 49, 194-207. [CrossRef]

49. The Good Scents Company. Available online: http://www.thegoodscentscompany.com/ (accessed on 5 February 2020).

50. Baenas, N.; Marhuenda, J.; García-Viguera, C.; Zafrilla, P.; Moreno, D.A.; Viguera, G. Influence of Cooking Methods on Glucosinolates and Isothiocyanates Content in Novel Cruciferous Foods. Foods 2019, 8, 257. [CrossRef]

51. Agneta, R.; Lelario, F.; De Maria, S.; Möllers, C.; Bufo, S.A.; Rivelli, A.R. Glucosinolate profile and distribution among plant tissues and phenological stages of field-grown horseradish. Phytochemistry 2014, 106, 178-187. [CrossRef]

52. Cools, K.; Terry, L.A. The effect of processing on the glucosinolate profile in mustard seed. Food Chem. 2018, 252, 343-348. [CrossRef]

53. Bell, L.; Concha, M.J.O.; Wagstaff, C. Identification and quantification of glucosinolate and flavonol compounds in rocket salad (Eruca sativa, Eruca vesicaria and Diplotaxis tenuifolia) by LC-MS: Highlighting the potential for improving nutritional value of rocket crops. Food Chem. 2014, 172, 852-861. [CrossRef]

54. Taranto, F.; Francese, G.; Di Dato, F.; D’Alessandro, A.; Greco, B.; Sanajà, V.O.; Pentangelo, A.; Mennella, G.; Tripodi, P. Leaf Metabolic, Genetic, and Morphophysiological Profiles of Cultivated and Wild Rocket Salad (Eruca and Diplotaxis Spp.). J. Agric. Food Chem. 2016, 64, 5824-5836. [CrossRef]

55. Borges, C.V.; Seabra, S.; Ponce, F.S.; Lima, G. Agronomic factors influencing Brassica productivity and phytochemical quality. In Brassica Germplasm—Characterization, Breeding and Utilization; El-Esawi, M.A., Ed.; IntechOpen: London, UK, 2018; pp. 57-74.

56. Fahey, J.W.; Zalcmann, A.T.; Talalay, P. The chemical diversity and distribution of glucosinolates and isothiocyanates among plants. Phytochemistry 2001, 56, 5-51. [CrossRef]

57. Bell, L.; Methven, L.; Signore, A.; Concha, M.J.O.; Wagstaff, C. Analysis of seven salad rocket (Eruca sativa) accessions: The relationships between sensory attributes and volatile and non-volatile compounds. Food Chem. 2017, 218, 181-191. [CrossRef] [PubMed]

(C) 2020 by the authors. Licensee MDPI, Basel, Switzerland. This article is an open access article distributed under the terms and conditions of the Creative Commons Attribution (CC BY) license (http://creativecommons.org/licenses/by/4.0/). 